\title{
Defining criteria to interpret multilocus variable- number tandem repeat analysis to aid Clostridium difficile outbreak investigation
}

\author{
Correspondence \\ George Broukhanski \\ george.broukhanski@oahpp.ca
}

Received 6 January 2011

Accepted 6 April 2011

\author{
George Broukhanski, ${ }^{1,2}$ Andrew Simor $^{1}$ and Dylan R. Pillai ${ }^{1,2}$ \\ ${ }^{1}$ Department of Laboratory Medicine \& Pathobiology, University of Toronto, Toronto, Ontario, \\ Canada \\ ${ }^{2}$ Ontario Agency for Health Protection and Promotion, Toronto, Ontario, Canada
}

\begin{abstract}
PFGE is currently the North American standard for surveillance for Clostridium difficile but lacks discriminatory power to aid outbreak investigation. A further limitation to PFGE is the high baseline rate of the epidemic North American pulsotype (NAP) 1 strain in hospitals. Multilocus variablenumber tandem repeat analysis (MLVA) appears to have superior discriminatory power but criteria to define clonality have not been set. We conducted surveillance for toxin-positive $C$. difficile infection (CDI) at a single academic health sciences centre between September 2009 and April 2010. Seventy-four patient specimens resulting in 86 discrete CDI episodes were subjected to PFGE and MLVA. Results were analysed using Bionumerics software to generate phylogenetic trees and coupled to patient demographic data. Amongst the NAP1 strains, two distinct clusters were identified by MLVA using $90 \%$ similarity as a cut-off by Manhattan distance-based clustering, four clusters using $95 \%$ and seven clusters using $97 \%$. Population analysis conducted on multiple colonies $(n=25)$ demonstrated that $1-3 \%$ difference in MLVA types was typical for a single individual. Typing was also conducted in the context of institutional outbreaks ( $n=42$, three outbreaks) in order to determine clusters within the NAP1 strain. By combining longitudinal surveillance with epidemiological information, single specimen population analysis and typing in the context of institutional outbreaks, we conclude that the use of the Manhattan distance-based clustering with a cut-off of $95-97 \%$ is capable of distinguishing outbreak clones from sporadic isolates.
\end{abstract}

\section{INTRODUCTION}

Clostridium difficile infection (CDI) is a major hospitalacquired infection in patients treated with antibiotics. Currently outbreak investigation of CDI in North America is performed using PFGE, and despite wide use there are factors which limit its utility due to a lack of discriminatory power in outbreak investigation, especially in geographical regions where baseline rates of the North American pulsotype (NAP) 1 prevalence are high (Pillai et al., 2010; Mulvey et al., 2010). For this reason, alternative typing methods might be better suited for this purpose. It was previously shown that multilocus variable-number tandem repeat analysis (MLVA) is a highly useful technique for investigation of outbreaks of $C$. difficile in hospitals (Marsh et al., 2006; Fawley et al., 2008; Goorhuis et al., 2009), especially in the case of clonal lineages, but criteria to distinguish outbreak from sporadic isolates are not yet

Abbreviations: CDI, Clostridium difficile infection; MLVA, multilocus variable-number tandem repeat analysis; NAP, North American pulsotype. defined. We assessed the utility of MLVA for hospital outbreak typing and propose criteria to define clusters during outbreaks. In this study, we use a combination of longitudinal surveillance of CDI, nosocomial outbreak typing and population analysis of stool specimens in order to validate cut-off values used to determine true clusters in the context of institutional outbreaks.

\section{METHODS}

Stool specimens $(n=86)$ were obtained from 74 consecutive patients with CDI at an academic health sciences centre over a period of 8 months (September 2009-April 2010). Specimens positive by Tox A/B II enzyme immunoassay (TechLab) were processed using ethanol for spore selection and plated on $C$. difficile moxalactam-norfloxacin $(\mathrm{CDMN})$ agar to grow under anaerobic conditions for $48 \mathrm{~h}$ (Aspinall \& Hutchinson, 1992). Grown colonies were tested with UV light, Gram staining and L-proline aminopeptidase test (PRO disk; Hardy Diagnostics) to confirm their identity. For PFGE typing, Peptone Yeast Glucose Broth (Anaerobe Systems) was inoculated with a single colony. PFGE was performed following standard procedures (Killgore et al., 2008). For MLVA, one colony from a CDMN plate was resuspended in $100 \mu \mathrm{l}$ InstaGene (Bio-Rad), heated at $100{ }^{\circ} \mathrm{C}$ for 
$10 \mathrm{~min}$, vortexed for $10 \mathrm{~s}$ and centrifuged at $8000 \mathrm{~g}$ for $2 \mathrm{~min}$. Two microlitres of the supernatant was used for amplification. A previously published MLVA protocol (van den Berg et al., 2007) was modified by eliminating $\mathrm{F}_{\mathrm{Cd}}$ and $\mathrm{H}_{\mathrm{Cd}}$ loci from analysis and modifying primer sequences to further accommodate sequence variation in $C$. difficile strains (Broukhanski et al., 2011). To aid identification of presumptive NAP1, NAP7 and NAP8 strains, the deletion within the $t c d C$ gene was detected by amplifying this region (Antikainen et al., 2009). PCR (total volume $8 \mu \mathrm{l}$ ) was performed using Type-it Microsatellite Master Mix (Qiagen). The cycling conditions were as follows: hot start at $90{ }^{\circ} \mathrm{C}$ for $5 \mathrm{~min}, 36$ cycles of $30 \mathrm{~s}$ at $90{ }^{\circ} \mathrm{C} / 60 \mathrm{~s}$ at $50{ }^{\circ} \mathrm{C} / 30 \mathrm{~s}$ at $72{ }^{\circ} \mathrm{C}$, with a final extension step of $30 \mathrm{~min}$ at $60{ }^{\circ} \mathrm{C}$. Amplicons were diluted $1: 20$ with distilled water and $1 \mu \mathrm{l}$ was transferred to $11 \mu \mathrm{l}$ formamide mixed with $0.1 \mu \mathrm{l}$ LIZ500 DNA ladder (Life Technologies) per tube. The plate was heated for $3 \mathrm{~min}$ at $95{ }^{\circ} \mathrm{C}$ and snap-cooled to $4{ }^{\circ} \mathrm{C}$. The Applied Biosystems 3130xl Genetic Analyzer (Life Technologies) was used to separate the amplicons and GENESCAN software (Life Technologies) was used to identify their length. Repeat numbers per locus were entered into BioNumerics software v. 6 (Applied Maths) to perform cluster analysis and coupled to patient demographic data, such as hospital unit location, date of admission and date of positive result by enzyme immunoassay. The Manhattan distance measure algorithm (a numerical coefficient which sums differences in repeat units and size of $t c d C$ deletion) and Ward clustering were used to generate the dendrograms (Ichikawa et al., 2010). Size of deletion in the $t c d C$ gene (18 bp in NAP1 and $38 \mathrm{bp}$ in NAP7/8 strains) was treated as a number of repeats for MLVA loci to allow reliable separation of highly pathogenic strains (NAP1/7/8) from other strains of lesser significance in outbreak investigations. To identify a level of diversity of $C$. difficile in a given outbreak and per specimen we used MLVA to type $C$. difficile isolates collected at three community hospitals with suspected outbreaks ( $n=12,14$ and 16) and also performed C. difficile population analysis by testing multiple colonies $(n=25)$ from specimens $(n=44)$ collected during surveillance in another hospital. These data were used to define criteria of interpretation of MLVA results for outbreak investigation.

\section{RESULTS}

For the hospital-based surveillance arm of this study, demographic breakdown revealed that the mean age of patients was 72 and $62 \%$ were females. Sixty-four patients $(86.5 \%)$ had single episodes with $10(14.5 \%)$ patients experiencing recurrent CDI. PFGE analysis demonstrated that $32 \mathrm{CDI}$ cases were due to the NAP1 pulsotype (37\%), $3(3.5 \%)$ due to NAP7, 2 (2.3\%) due to NAP4, 1 (1.2\%) due to NAP2, with the remainder (56\%) due to arbitrarily assigned pulsotypes. Typing with MLVA demonstrated variability among NAP1 strains (Fig. 1). Two clusters of NAP1 could be identified when $90 \%$ similarity was used as a cut-off by Manhattan distance-based clustering, four clusters when $95 \%$ similarity was used as a cut-off and seven clusters when $97 \%$ was used as a cut-off. MLVA of specimens from three other hospital outbreaks (Fig. 2) was used to evaluate the degree of variation of MLVA profiles in isolated C. difficile strains. These results showed that the degree of heterogeneity of MLVA types within an outbreak varies by hospital. Nevertheless, observed clusters in these three outbreaks had MLVA profiles with less than $5 \%$ difference. Typing multiple colonies per specimen (population analysis) with MLVA demonstrated that usually $(90 \%, n=39)$ the diversity of strains is minimal $(2-3 \%$ difference using the Manhattan distance measure) and is limited to one locus (most often $\mathrm{A} 6_{\mathrm{Cd}}$ or $\mathrm{C6}_{\mathrm{Cd}}$ ). Only in one specimen did all 25 colonies have an identical MLVA profile. Few specimens $(<10 \%, n=4)$ had strains with a difference in more than one locus, which, most likely, represent polyclonal infections (Fig. 3). Clustering analysis of MLVA data of all isolates collected over the surveillance

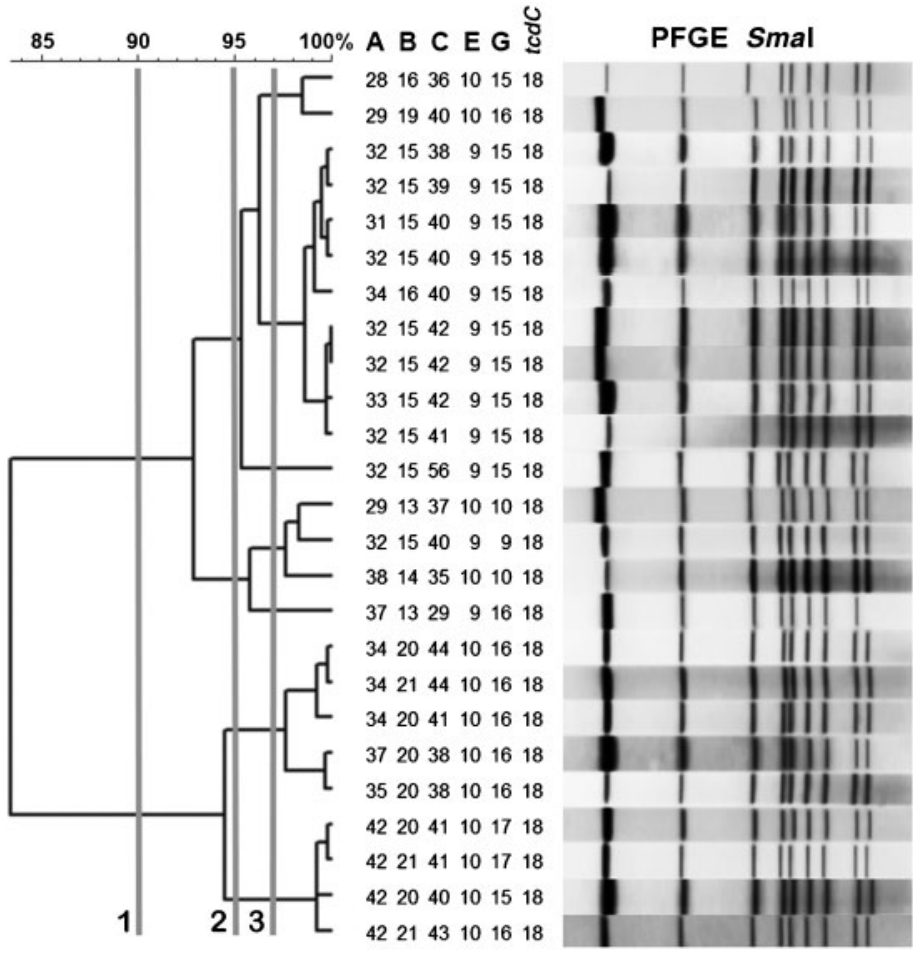

Fig. 1. Comparison of PFGE (right) and MLVA (left) for NAP1 clinical isolates. The numerical values represent repeat units at five separate loci based on the MLVA sequencing method, except for the final value, which represents the $18 \mathrm{bp}$ deletion in the $t c d C$ gene found in NAP isolates. The vertical lines represent three cutoff values at $15 \%, 9 \%$ and $3 \%$ difference based on the Manhattan distance algorithm. 

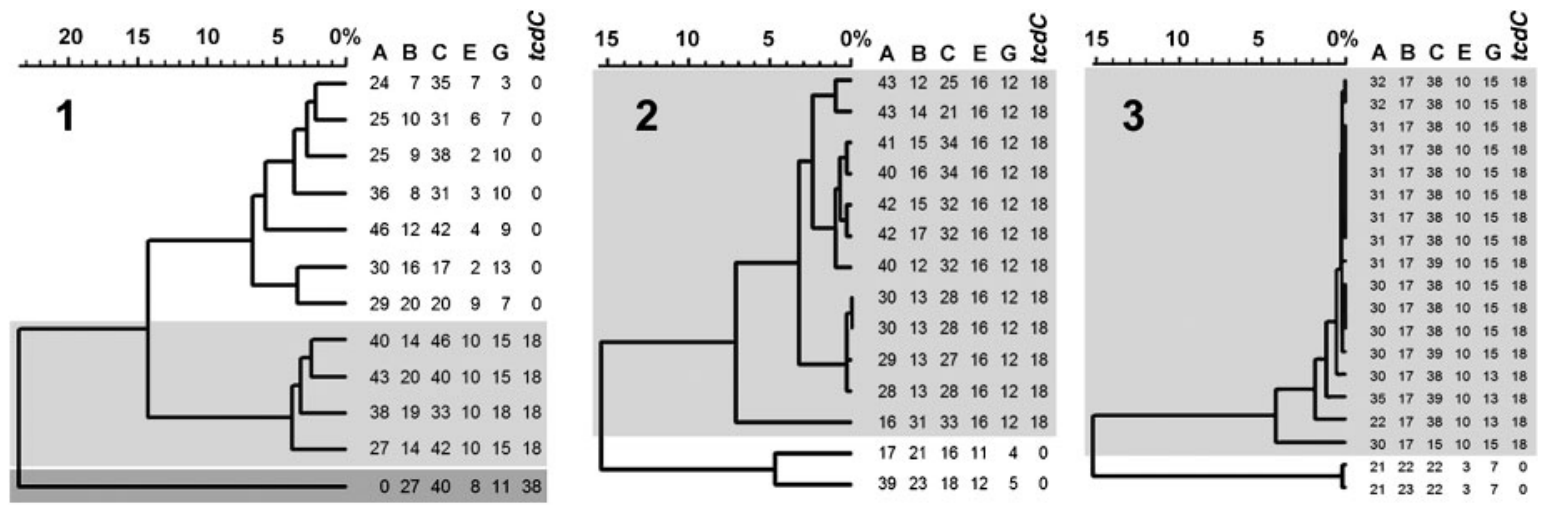

Fig. 2. MLVA of specimens from three hospital outbreaks. NAP1 (grey) and NAP7 (dark-grey) strains are indicated using shadowing. Three types of hospital outbreaks were identified: a mixture of NAP1 and non-NAP1 isolates (1); heterogeneous NAP1 isolates (2); and homogeneous NAP1 isolates (3).

period shows two well-defined groups of NAP1 strains (Fig. 4). In an attempt to identify whether any demographic parameters can be linked to these groups, we paired MLVA results with patient demographic data. The best match was observed when MLVA data were coupled with the date of admission. Two MLVA clusters using a $5 \%$ cut-off based on the Manhattan distance-based clustering correlated with admission in January and March (Fig. 5).

\section{DISCUSSION}

The optimal method of typing $C$. difficile strains in the context of institutional outbreaks has yet to be defined. PFGE and ribotyping are the two methods used most commonly in North America and Europe, respectively. Other typing methods such as restriction endonuclease analysis, toxinotyping and multilocus sequence typing are used most often for research purposes. MLVA was identified as the most discriminatory typing method (Kuijper et al., 2009), and when seven typing techniques were compared, it was shown that MLVA and restriction endonuclease analysis are the only methods suitable for outbreaks investigation (Killgore et al., 2008). However, MLVA is thought to be too discriminatory for this purpose and its ability to identify multiple MLVA types within a single specimen could obscure epidemiological links if only one isolate per specimen is typed (Tanner et al., 2010). Therefore, the challenge is to define cut-offs that empower MLVA to define clusters within the context of hospital outbreaks. We show here that changing the cut-off values from $90 \%$ to $97 \%$ based on the Manhattan distance-based clustering results in the identification of two or seven clusters, respectively (Fig. 1). Hence, the cut-off value chosen can dramatically alter the interpretation of clonality.

It is known that the $C$. difficile genome is highly plastic (Sebaihia et al., 2006; Scaria et al., 2010) and therefore overly discriminatory cut-off values may exaggerate differences between clinical isolates. To help identify what is the most appropriate cut-off value, we analysed MLVA profiles among specimens collected during institutional outbreaks where presumably a single clone was spreading

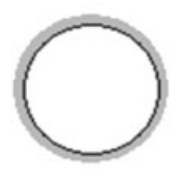

1

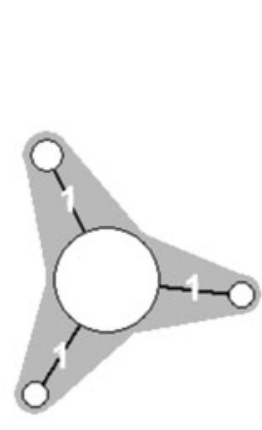

2

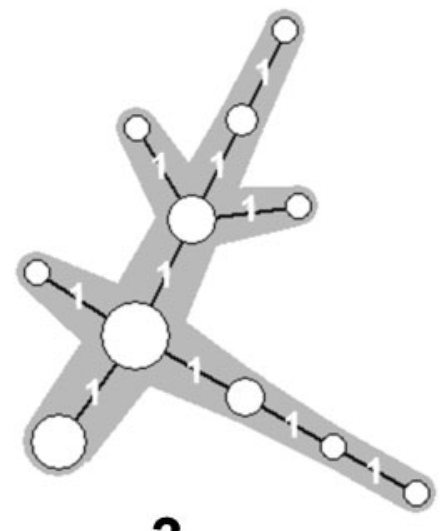

3
Fig. 3. Minimum spanning trees of MLVA profiles of $C$. difficile colonies $(n=25)$ isolated from three representative clinical specimens are depicted. Three types of $\mathrm{C}$. difficile populations within a specimen were identified: monoclonal ( $1,2 \%$ of all specimens); low diversity (2, $90 \%$ of specimens); and high diversity (3, $8 \%$ of specimens). Neighbouring nodes differ from each other by a single locus based on the MLVA method. A total of 44 specimens from a single institution were analysed in this way. 


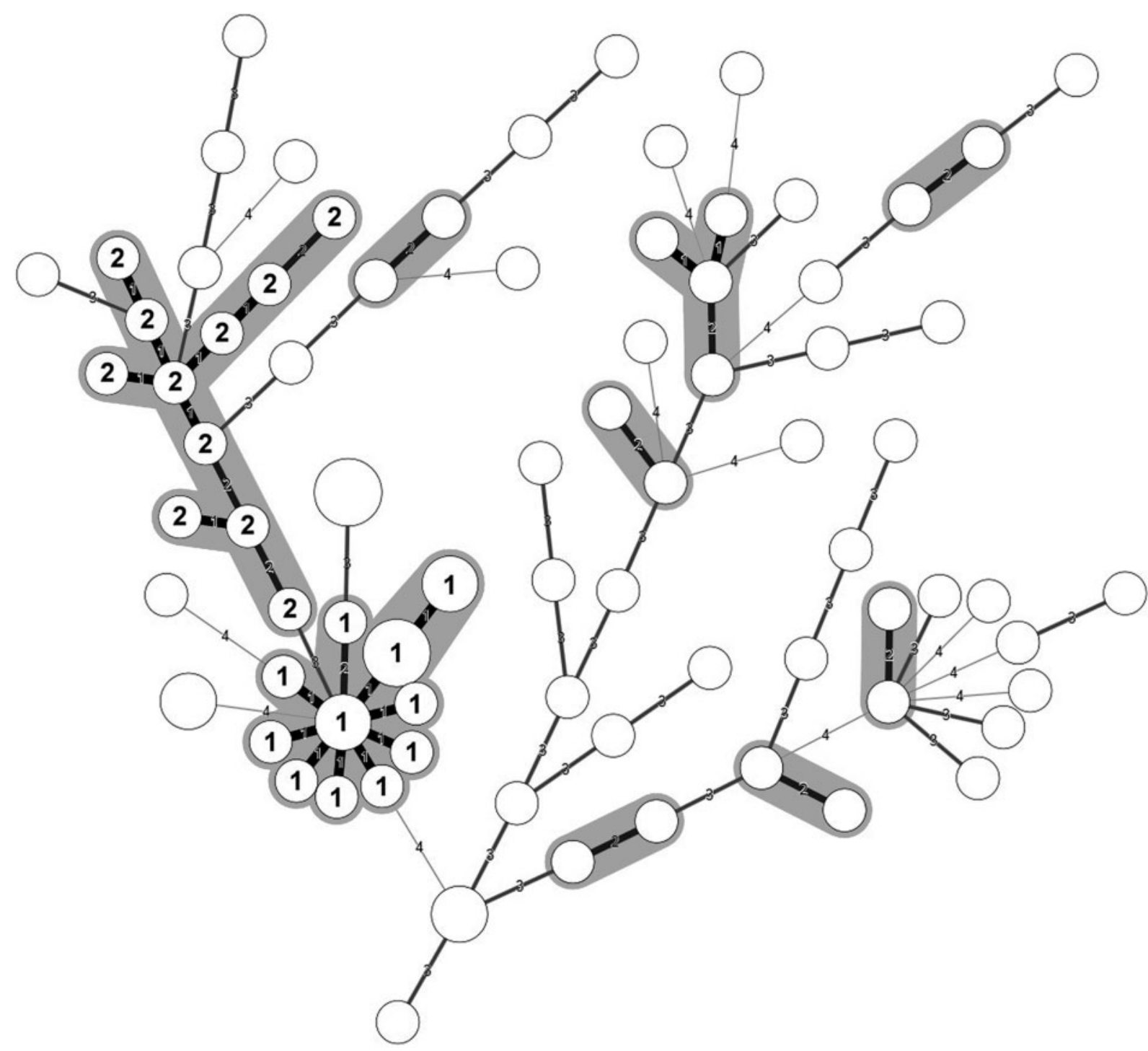

Fig. 4. Clustering analysis based on MLVA typing of clinical isolates collected over a period of 8 months in a hospital. Isolates within the grey shadowed area are different to each other at $\leqslant$ two loci. Two clusters of NAP1 strains were identified as indicated by nodes labelled with 1 or 2 .

in the hospital. Results of this study, performed on specimens from three representative hospitals, demonstrate that three possible MLVA patterns can be observed (Fig. 2). In those sets of specimens, when a single 'clone' was present during the outbreak based on epidemiological information, natural variation was observed in the range of $3-5 \%$ within the outbreak cluster. Results of population analysis of multiple isolates from a single specimen demonstrated that the majority of specimens ( $90 \%)$ have variation in one or two loci (especially $\mathrm{A} 6_{\mathrm{Cd}}$ and $\mathrm{C6}_{\mathrm{Cd}}$ ). This variation was approximately $1-3 \%$ difference based on the Manhattan distance-based clustering, suggesting that the cut-off value should not be less than $3 \%$. Combination of these data (MLVA of hospital isolates and population analysis of multiple isolates per specimen) indicates that a cut-off value greater than $3 \%$ but less than $5 \%$ based on the Manhattan distance-based clustering identifies true outbreak clones. Using this cut-off value range, MLVA typing was used during longitudinal hospital surveillance and, when coupled to epidemiological information, was able to define clusters that correlated with the date of admission thus validating the interpretive criteria proposed here (Fig. 5). These results will enable infection control investigators to distinguish outbreak clones from sporadic isolates during a hospital outbreak.

\section{Conclusions}

MLVA is a highly discriminatory typing method capable of defining clusters of NAP1 isolates when the correct cut-off 


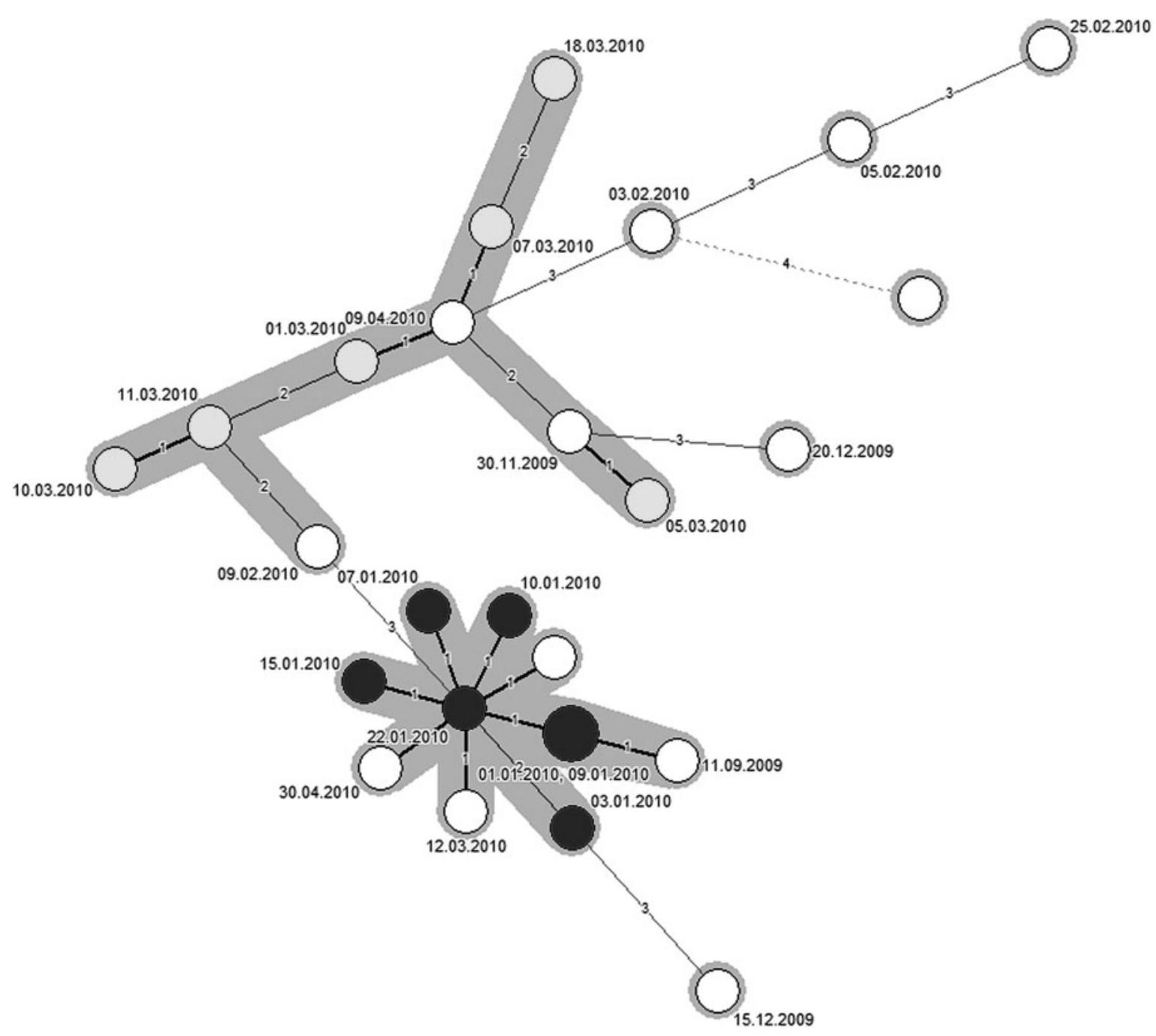

Fig. 5. Minimum spanning tree based on MLVA typing of NAP1 isolates coupled to the date of admission. Isolates from January (dark-grey) and March (grey) are compared to those from other months (white).

values are used. Population analysis suggests that a cut-off value of $<3 \%$ based on the Manhattan distance-based clustering is overly discriminatory due to the natural variation of $C$. difficile genotypes in a single specimen, but a cut-off of 3-5\% should capture true clusters based on the five loci we have used. Hospital surveillance using clinical and epidemiological data reinforces that a cut-off of 3-5\% is able to identify true clusters of NAP1. Further multicentre studies relying on both MLVA data and clinical epidemiological information are required to validate the 3 $5 \%$ cut-off value proposed here.

\section{ACKNOWLEDGEMENTS}

We thank the medical laboratory technicians in the Molecular Surveillance department at the Public Health Laboratory in Toronto for their expert technical assistance. We thank the participating institutions for their active role in C. difficile surveillance. We also thank Kate Henbest for technical assistance.

\section{REFERENCES}

Antikainen, J., Pasanen, T., Mero, S., Tarkka, E., Kirveskari, J., Kotila, S., Mentula, S., Könönen, E., Virolainen-Julkunen, A. R. \& other authors (2009). Detection of virulence genes of Clostridium difficile by multiplex PCR. APMIS 117, 607-613.

Aspinall, S. T. \& Hutchinson, D. N. (1992). New selective medium for isolating Clostridium difficile from faeces. J Clin Pathol 45, 812-814.

Broukhanski, G., Low, D. E. \& Pillai, D. R. (2011). Modified multiplelocus variable-number tandem-repeat analysis for rapid identification and typing of Clostridium difficile during institutional outbreaks. J Clin Microbiol 49, 1983-1986.

Fawley, W. N., Freeman, J., Smith, C., Harmanus, C., van den Berg, R. J., Kuijper, E. J. \& Wilcox, M. H. (2008). Use of highly discriminatory fingerprinting to analyze clusters of Clostridium difficile infection cases due to epidemic ribotype 027 strains. J Clin Microbiol 46, 954960.

Goorhuis, A., Legaria, M. C., van den Berg, R. J., Harmanus, C., Klaassen, C. H., Brazier, J. S., Lumelsky, G. \& Kuijper, E. J. (2009). Application of multiple-locus variable-number tandem-repeat ana- 
lysis to determine clonal spread of toxin A-negative Clostridium difficile in a general hospital in Buenos Aires, Argentina. Clin Microbiol Infect 15, 1080-1086.

Ichikawa, K., Yagi, T., Inagaki, T., Moriyama, M., Nakagawa, T., Uchiya, K., Nikai, T. \& Ogawa, K. (2010). Molecular typing of Mycobacterium intracellulare using multilocus variable-number of tandem-repeat analysis: identification of loci and analysis of clinical isolates. Microbiology 156, 496-504.

Killgore, G., Thompson, A., Johnson, S., Brazier, J., Kuijper, E., Pepin, J., Frost, E. H., Savelkoul, P., Nicholson, B. \& other authors (2008). Comparison of seven techniques for typing international epidemic strains of Clostridium difficile: restriction endonuclease analysis, pulsed-field gel electrophoresis, PCR-ribotyping, multilocus sequence typing, multilocus variable-number tandem-repeat analysis, amplified fragment length polymorphism, and surface layer protein A gene sequence typing. J Clin Microbiol 46, 431-437.

Kuijper, E. J., van den Berg, R. J. \& Brazier, J. S. (2009). Comparison of molecular typing methods applied to Clostridium difficile. Methods Mol Biol 551, 159-171.

Marsh, J. W., O'Leary, M. M., Shutt, K. A., Pasculle, A. W., Johnson, S., Gerding, D. N., Muto, C. A. \& Harrison, L. H. (2006). Multilocus variablenumber tandem-repeat analysis for investigation of Clostridium difficile transmission in hospitals. J Clin Microbiol 44, 2558-2566.
Mulvey, M. R., Boyd, D. A., Gravel, D., Hutchinson, J., Kelly, S., McGeer, A., Moore, D., Simor, A., Suh, K. N. \& other authors (2010). Hypervirulent Clostridium difficile strains in hospitalized patients, Canada. Emerg Infect Dis 16, 678-681.

Pillai, D. R., Longtin, J. \& Low, D. E. (2010). Surveillance data on outbreaks of Clostridium difficile infection in Ontario, Canada, in 2008-2009. Clin Infect Dis 50, 1685-1686, author reply 1686.

Scaria, J., Ponnala, L., Janvilisri, T., Yan, W., Mueller, L. A. \& Chang, Y. F. (2010). Analysis of ultra low genome conservation in Clostridium difficile. PLoS ONE 5, e15147.

Sebaihia, M., Wren, B. W., Mullany, P., Fairweather, N. F., Minton, N., Stabler, R., Thomson, N. R., Roberts, A. P., Cerdeño-Tárraga, A. M. \& other authors (2006). The multidrug-resistant human pathogen Clostridium difficile has a highly mobile, mosaic genome. Nat Genet 38, 779-786.

Tanner, H. E., Hardy, K. J. \& Hawkey, P. M. (2010). Coexistence of multiple multilocus variable-number tandem-repeat analysis subtypes of Clostridium difficile PCR ribotype 027 strains within fecal specimens. J Clin Microbiol 48, 985-987.

van den Berg, R. J., Schaap, I., Templeton, K. E., Klaassen, C. H. \& Kuijper, E. J. (2007). Typing and subtyping of Clostridium difficile isolates by using multiple-locus variable-number tandem-repeat analysis. J Clin Microbiol 45, 1024-1028. 